\title{
Dynamic interaction between domain walls and nanowire vertices
}

\author{
D. M. Burn, ${ }^{*}$ M. Chadha, S. K. Walton, and W. R. Branford \\ Department of Physics, Prince Consort Road, Imperial College London, London SW7 2BZ, United Kingdom
}

(Received 23 July 2014; revised manuscript received 26 September 2014; published 13 October 2014)

\begin{abstract}
The dynamic behavior of magnetic domain walls incident upon a Y-shaped junction between three nanowires is explored. Details in the micromagnetic structure of the domain wall and the local spin texture at a vertex result in a complex interaction which ultimately determines the propagation direction of the domain wall following the vertex. This interaction has been explored through both magneto-optical Kerr effect experiments and micromagnetic simulations on single-vertex structures. Differences in the micromagnetic structure of incident domain walls in the dynamic regime have a significant influence on the interaction. We observe an oscillatory path selection with increasing domain wall propagation distance preceding the vertex. Our analysis shows that this behavior originates from cyclic reversals of the transverse domain wall chirality due to Walker breakdown processes.
\end{abstract}

DOI: 10.1103/PhysRevB.90.144414

PACS number(s): 75.78.Fg, 75.60.Ch

\section{INTRODUCTION}

The behavior of domain walls (DWs) in magnetic nanostructures is of fundamental scientific interest as studies of these simplified systems give us a greater understanding of the magnetic and magnetization reversal processes in many magnetic materials. Additionally, much research interest in this area is driven by the potential for novel technological developments such as logic [1] and memory [2] devices. More recently the ability to detect [3] and manipulate [4-8] magnetic nanoparticles with DWs has been demonstrated with possible futures in lab-on-a-chip technologies.

Understanding of the interactions between the DW's internal micromagnetic structure and nanoscale geometrical structuring is an important step towards realizing these technologies. Extensive work covers the field manipulation of DWs along nanowires, detailed analysis of their internal micromagnetic structure showing different DW types and chiralities [9] and how different DWs interact with geometrical structural features $[10,11]$.

Previous studies on the behavior of DWs at junctions between nanowires have linked the pinning at a cross-shaped vertex with DW internal micromagnetic structure [12,13]. In $120^{\circ}$ vertices a similar interaction leads to a source of asymmetry in the path of DWs as they depin from the vertex [14] and for vortex walls at junctions with $60^{\circ}$ openings, topological edge defects in the micromagnetic DW structure have been suggested as a way to determine the path of a DW at a junction [15]. The behavior of magnetic DWs at junctions is also important in artificial spin ice systems with $120^{\circ}$ vertices where the nucleation and propagation of magnetic domain walls enable magnetic charges to migrate around the system $[16,17]$. DW chirality has been identified as a source of selectivity in the direction of chains of reversed nanobars in these systems [18]. Other recent work has focused on the dynamics of propagating DWs [19-21] where, beyond a critical Walker field, periodic changes in DW micromagnetic structure from Walker breakdown occur [22,23]. These lead to more complex interactions with nanostructure geometry $[24,25]$ and

\footnotetext{
*d.burn@imperial.ac.uk
}

require additional consideration beyond many of the models based on the quasistatic understanding of magnetic DWs.

The work here examines the behavior of magnetic DWs in the dynamic regime as they interact with $\mathrm{Y}$-shaped vertex structures between three nanowires with $120^{\circ}$ separation. The effect of time-dependent changes in DW micromagnetic structure upon the DW propagation path following the interaction is investigated.

\section{EXPERIMENT}

To investigate the interaction between DWs and nanowire vertices, individual vertex structures were prepared as shown in Fig. 1. Structures consisted of a vertex connecting three 100-nm-wide, 15- and 20-nm-thick planar nanowires with a $120^{\circ}$ angular separation. The length, $L$, of the horizontal arm was varied from 0.5 to $25 \mu \mathrm{m}$ and included a hook structure with a $500 \mathrm{~nm}$ radius for the injection of transverse DWs [26]. The sense of rotation of the hook, and the applied field sequence determine the DW chirality, giving an "up chirality" DW from the "down hook" structure. This chirality relates to the orthogonal magnetization component within the DW structure as shown by the dark contrast on the outer edge of the hook structure in the magnetic force microscope image in Fig. 1(d). The two nanowires following the vertex were $20 \mu \mathrm{m}$ long and were terminated with $4-\mu \mathrm{m}$-long tapered ends to restrict unwanted DW nucleation.

Structures were prepared on $\mathrm{Si} / \mathrm{SiO}_{2}$ substrates by electron beam lithography followed by thermal evaporation and lift-off of permalloy, deposited from a single powdered alloy source of nominal composition $\mathrm{Ni}_{81} \mathrm{Fe}_{19}$. The deposition thickness was monitored by an in situ quartz rate monitor and later confirmed by atomic force microscopy. Focused longitudinal magneto-optical Kerr effect (MOKE) measurements probed the magnetization reversal behavior from a $\sim 5 \mu \mathrm{m}$ laser spot projected onto the sample surface. This was positioned, in turn, on the individual nanowires in the structure at the positions indicated by the ellipses in Fig. 1. Magnetization reversal was driven by a $430 \mathrm{Oe}, 4 \mathrm{~Hz}$ sinusoidal field applied along the axis of the horizontal nanowire. A smaller additional 8 Oe sinusoidal field was applied perpendicular to the horizontal nanowire with $\mathrm{a} \pm \pi / 2$ phase difference to provide an effective 


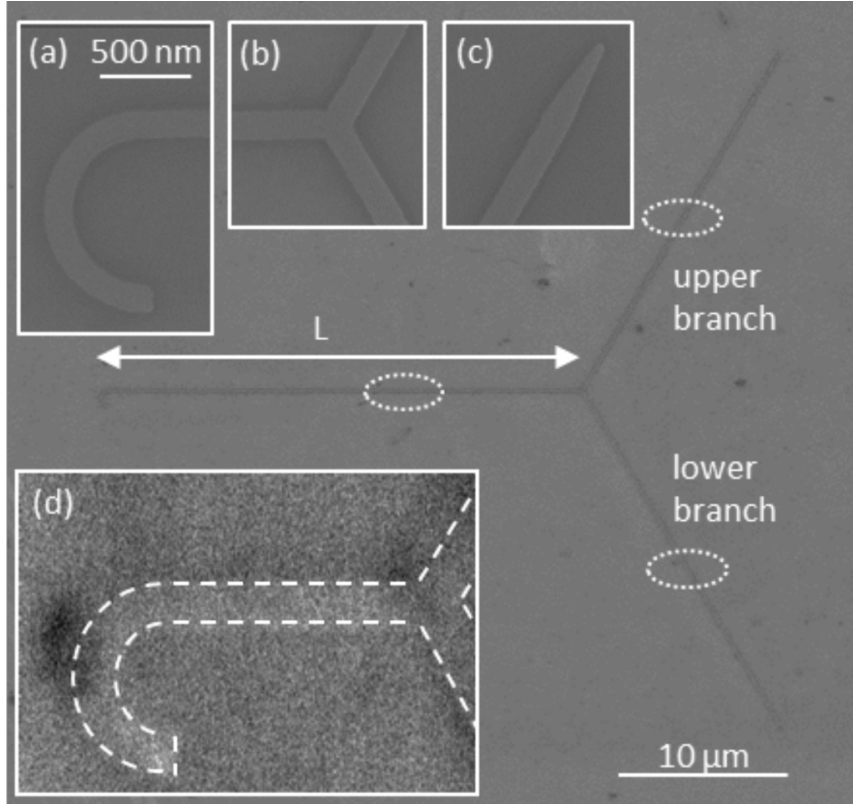

FIG. 1. Scanning electron microscope image of the fabricated structures comprising a hook structure (a) separated from a $120^{\circ}$ vertex (b) by a nanowire of length $L$. MOKE measurement positions are indicated by dotted ellipses. The insets show higher magnification images of the (a) hook, (b) vertex, and (c) tapered ends of the structures. (d) Magnetic force microscopy shows the injection of a domain wall from the hook structure.

rotating elliptical field for the injection of known chirality DWs from the hook structures [26]. The Kerr signal was averaged over hundreds of field cycles and shows the variation in the component of magnetization as a function of the applied magnetic field along the horizontal axis.

Experimental results are supported by micromagnetic simulations performed in OOMMF (Object Oriented MicroMagnetic Framework) [27]. The simulations were based on 100-nmwide, 10-nm-thick nanowire structures connected at a $120^{\circ}$ vertex. A $5 \times 5 \times 10 \mathrm{~nm}$ mesh and typical micromagnetic parameters for permalloy, i.e., $M_{S}=860 \times 10^{3} \mathrm{~A} / \mathrm{m}, A=$ $13 \times 10^{-12} \mathrm{~J} / \mathrm{m}$, and zero magnetocrystalline anisotropy were used.

The field-dependence to the DW-vertex interaction was examined with $0.5-\mu \mathrm{m}$-long wires and an artificially high Gilbert damping parameter of $\alpha=0.5$, reasonable in this quasistatic regime [28]. The initial magnetization of the system was based on a remanent state with magnetization in the negative $x$ direction along the horizontal bar and a head-to-head transverse DW structure with preconditioned chirality was included in the horizontal arm. An axial field was applied in 1 Oe steps until the magnetization in all three nanowires had reversed.

Additional simulations were performed on similar structures in the dynamic regime using a more realistic damping parameter of $\alpha=0.01$. In this case the horizontal arm was extended to $1 \mu \mathrm{m}$ allowing for variation of the initial DWvertex separation. An axial applied field of fixed magnitude was applied and the magnetization state of the system was recorded as a function of time.
In both cases the magnetostatic effects due to the end of the horizontal nanowire were removed through the inclusion of a plate of fixed spins at the nanowire ends [29]. The other two wires were sufficiently long that the end effects were not significant in influencing the DW interaction at the vertex.

\section{RESULTS AND DISCUSSION}

MOKE hysteresis loops shown in Fig. 2 demonstrate the typical ferromagnetic behavior of the $\mathrm{NiFe}$ nanowires. The figure here shows results from the horizontal bar of a 20-nm-thick structure with $L=20 \mu \mathrm{m}$. The two loops show dramatically different switching fields which are obtained when the rotation direction of the applied field is reversed. The switching at $\approx 435$ Oe with the anticlockwise rotating field represents the high field required for the direct reversal of the magnetization within a nanowire of this geometry. In this case the rotation of the field and sense of the hook do not lead to the injection of a DW into the nanowire. However, with clockwise rotating fields, the switching field of the bar reduces to $H_{\mathrm{INJ}} \approx 85$ Oe. This field represents the injection of a DW with up chirality from the hook structure into the horizontal nanowire. The magnetization in the bar then reverses, mediated by the propagation of the DW.

Figure 3 shows further hysteresis loops, this time measured in the upper branch of the structure. Again an up chirality DW is injected into the horizontal bar from a down hook structure with a clockwise rotating field. The three loops in the figure correspond to different hook-vertex separations, $L$. For $L=1.4 \mu \mathrm{m}$ in Fig. 3(a) the magnetization reversal takes place at a field $H_{C 1}=90 \mathrm{Oe}$, but with a shorter $L=1.1 \mu \mathrm{m}$ structure in Fig. 3(b), reversal takes place at a higher field of $H_{C 2}=130$ Oe. Figure 3(c) shows the behavior for a $L=0.7 \mu \mathrm{m}$ structure with a more complex step shape to the $M H$ loop which is found in a minority of cases. This can be explained due to the averaging of the MOKE system over many field cycles, combining the hysteresis loops with a switching field at $H_{C 2}$

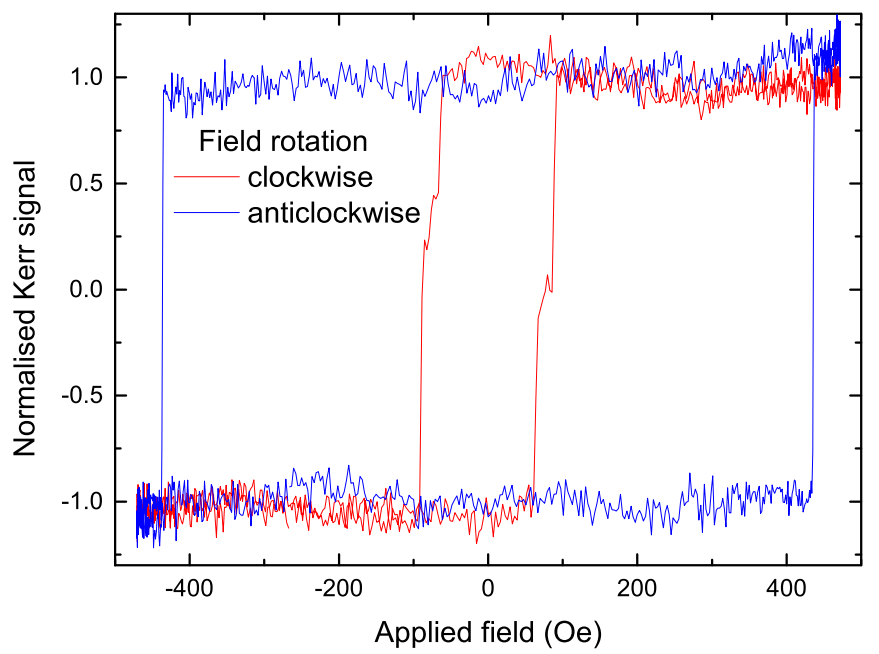

FIG. 2. (Color online) Magnetization as a function of field showing hysteresis loops for the $20-\mu \mathrm{m}$-long horizontal bar of a $20-\mathrm{nm}$ thick structure with a down hook. Two loops are shown when the field is rotated in the clockwise and anticlockwise directions. 


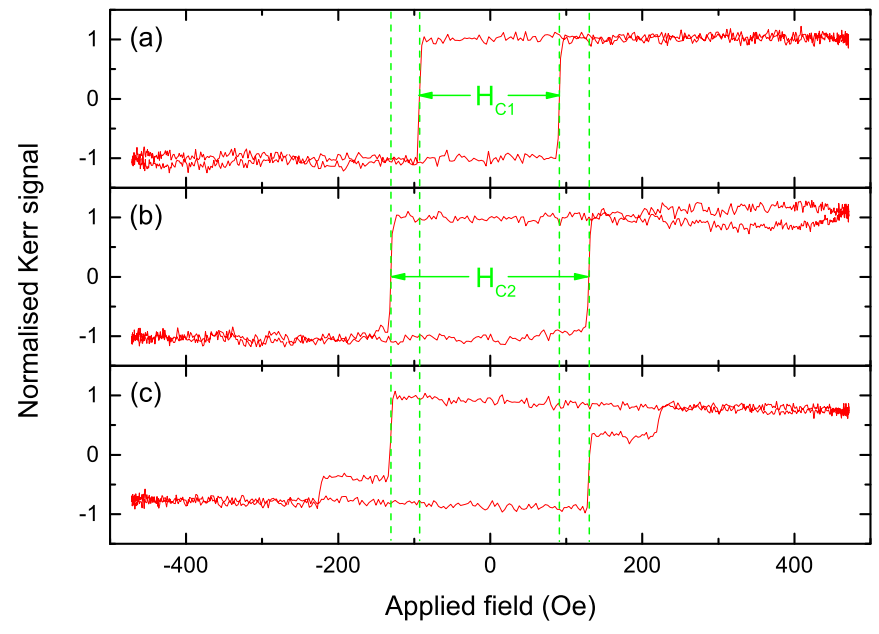

FIG. 3. (Color online) Hysteresis loops from the upper branch of a hook down structure with clockwise rotating fields. Three loops correspond to different lengths, $L$, of the horizontal bar: (a) $1.4 \mu \mathrm{m}$, (b) $1.1 \mu \mathrm{m}$, and (c) $0.7 \mu \mathrm{m}$.

with others, where reversal does not take place until a much higher field (220 Oe).

The two separate reversal fields, $H_{C 1}$ and $H_{C 2}$, can be explained by the interaction and subsequent propagation of the DW through the vertex structure. The DW is injected into the horizontal bar at $H_{\mathrm{INJ}}$ and propagates towards the vertex end of the bar, reversing the magnetization in the horizontal bar. When the field is in excess of $H_{C 1}$ the DW is able to continue propagation along one of the branches of the Y-shaped structure following the interaction with the vertex. For the $L=1.4 \mu \mathrm{m}$ structure in Fig. 3(a) the DW propagates along the upper branch resulting in the magnetization reversal at $H_{C 1}$ in that branch. However, for the $L=1.1 \mu \mathrm{m}$ structure in Fig. 3(b) the DW propagates along the lower branch so no change in magnetization is measured at $H_{C 1}$ in the upper branch. Instead, the magnetization in the upper branch reverses due to the nucleation and injection of a secondary DW from the vertex at a higher field, $H_{C 2}$. Therefore the measurement of the reversal field in either branch allows the path of the DW to be determined as it depins from the Y-shaped vertex. For the 20-nmthick structures in Fig. 3, $H_{C 1}$ is comparable to $H_{\mathrm{INJ}}$ so it may represent an upper bound to the propagation field as the value measured may be limited by the injection of the initial DW.

The step in the hysteresis loop in Fig. 3(c) shows an additional process taking place at higher fields which can be attributed to the unsuccessful injection of the initial DW into the horizontal bar. Magnetization reversal in the branch following the $\mathrm{Y}$-shaped vertex is then measured at a higher field due to the nucleation of a DW at the vertex. This process differs from the nucleation of a secondary DW at $H_{C 2}$ as the nucleation occurs before the reversal in the other two branches. This difference leads to the increased field for this process.

Micromagnetic simulations on nanowire vertex structures support this interpretation and offer additional insight into the underlying physics involved in the DW-vertex interaction. Figures 4(a) and 4(b) show the evolution of the micromagnetic spin structure as a function of applied field for an initial DW in the horizontal bar with up and down chirality, respectively. In

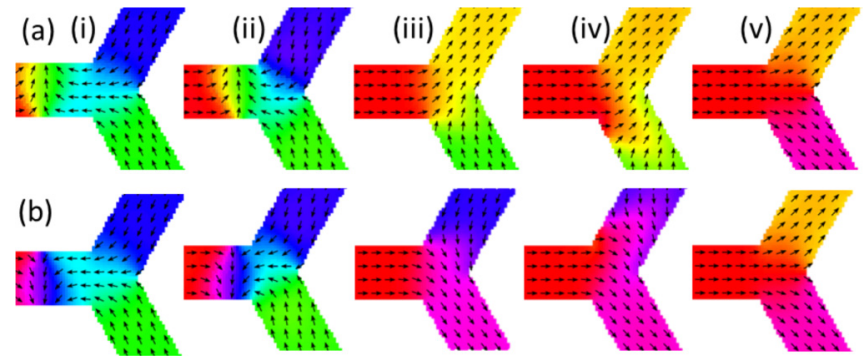

FIG. 4. (Color online) Micromagnetic simulations showing the interaction between a DW with (a) up and (b) down chirality with a nanowire vertex. The equilibrium state is shown for several fields: (i) $50 \mathrm{Oe}$, (ii) $180 \mathrm{Oe}$, (iii) $190 \mathrm{Oe}$, (iv) $370 \mathrm{Oe}$, and (v) $380 \mathrm{Oe}$.

both cases the DW approaches the vertex as the increasing field raises the Zeeman energy which competes with the repulsion between the magnetically charged DW and vertex. Beyond a certain field, $H_{C 1}$, Fig. 4(iii) shows the DW depins from the vertex and propagates along one of the nanowire branches. A further increase in field leads to the assisted nucleation of a secondary DW at the vertex. This is then injected into the other branch at a higher field, $H_{C 2}$, leading to the reversal of the magnetization in that branch shown in Fig. 4(v).

The micromagnetic simulations show that the chirality of the incident DW determines which branch the DW takes as it depins from the vertex. This preference originates from the component of magnetization in the DW that is perpendicular to the wire axis and leads to breaking of the symmetry of the system. This component of magnetization favors the magnetization orientation in one of the two angled branches and leads to the up chirality DW preferentially taking the upper path at the vertex and the down chirality DW taking the lower branch.

Importantly, the experimental results show the reversal behavior at both $H_{C 1}$ and $H_{C 2}$ despite an additional $8 \mathrm{Oe}$ symmetry-breaking perpendicular field component which is required for the DW injection from the hook structures. This shows that the DW chirality is having a stronger influence on the DW path than the additional undesirable field.

In Fig. 3 the magnetization reversal takes place at either $H_{C 1}$ or $H_{C 2}$ depending on the length of the horizontal bar between the injection hook structure and the vertex. This length dependence is shown more clearly in Fig. 5 where the switching fields for the upper branch of a structure with a down hook is shown as a function of hook-vertex separation. These results have been extracted from many hysteresis loops measured on both (a) 20- and (b) 15-nm-thick structures. Both figures show the two discrete switching fields $H_{C 1}$ and $H_{C 2}$ describing the path of the DWs at the vertex, and a periodic dependence between the two indicated by the shaded regions.

In Fig. 5(a) the switching field measured in the top branch of a 20-nm-thick structure alternates between the two switching field values of $H_{C 1}=90 \mathrm{Oe}$ and $H_{C 2}=130 \mathrm{Oe}$. Between $L=1.2$ and $1.7 \mu \mathrm{m}$ switching at $H_{C 1}$ shows the DW took the upper branch while for $L=0.6-1.1 \mu \mathrm{m}$ switching at $H_{C 2}$ shows the DW took the lower branch. This change in path direction is periodic and linked to the hook-vertex separation distance. Additionally, higher field reversal events are found from step-shaped hysteresis loops in some cases, representing the unsuccessful injection of a DW into the horizontal 


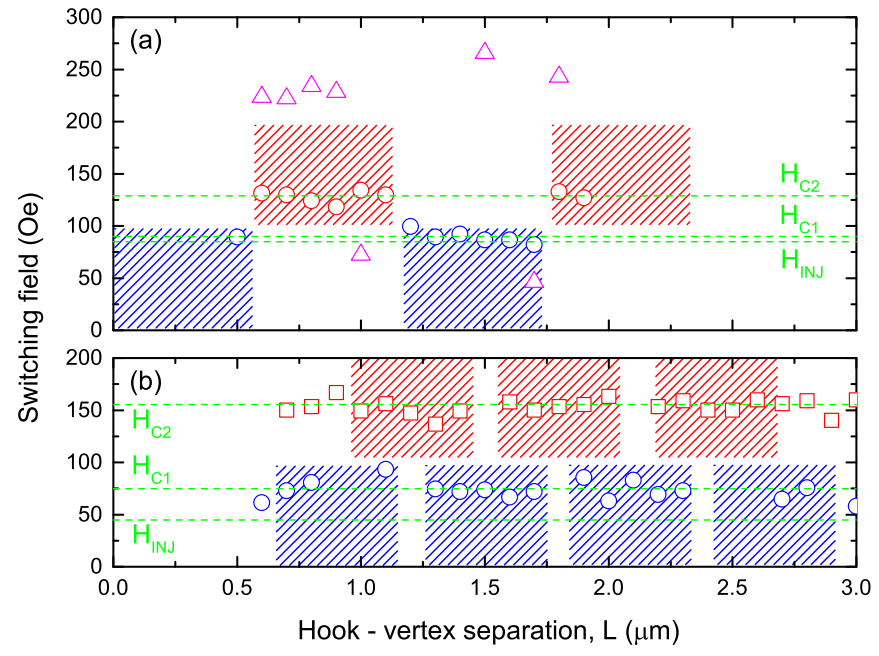

FIG. 5. (Color online) Switching field of the upper branch of a (a) 20- and (b) 15-nm-thick hook down structure in a clockwise rotating field as a function of hook-vertex separation. The dashed lines show the DW injection field, $H_{\mathrm{INJ}}$, and switching fields at $H_{C 1}$ and $H_{C 2}$ corresponding to reversal from the transmission of the incident DW or nucleation of a secondary DW, respectively. Shaded regions highlight the periodic nature of the data.

bar. With the 15-nm-thick structures, Fig. 5(b) shows more complex behavior where the majority of hysteresis loops show similar step-shaped behavior representing magnetization reversal events at both $H_{C 1}=75 \mathrm{Oe}$ and $H_{C 2}=155 \mathrm{Oe}$. Again these steps arise due to the averaging of the two reversal fields over many field cycles. However, in some cases, reversal behavior purely at $H_{C 1}$ or $H_{C 2}$ was measured, corresponding to the gaps between the shaded regions which demonstrate the periodicity. In these thinner structures the injection field was $H_{\mathrm{INJ}}=45 \mathrm{Oe}$, significantly lower than both $H_{C 1}$ and $H_{C 2}$ so in this case it is unlikely that the DW injection is limiting the propagation process at the vertex.

The periodic behavior shown in Fig. 5 suggests that the path of the DW as it propagates through the vertex alternates between the upper and lower branches. From the micromagnetic simulations this can be understood as periodic changes in DW chirality which take place before the interaction with the vertex. These arise due to Walker breakdown effects on the DW chirality as it propagates along the horizontal branch [22,23].

The Walker breakdown process results in periodic changes in the DW chirality with fields in excess of the Walker field (typically 15 Oe [30]). Here the fast motion of a transverse DW is interrupted by the formation of an antivortex core at the pinching end of the DW. This traverses the wire width, reversing the wall chirality before annihilating at the opposite edge of the wire. This process repeats, leading to periodic changes in the DW chirality during its motion along a wire, and when combined with the chirality-dependent selectivity at the vertex gives an explanation for the periodic dependence of the DW path as a function of hook-vertex separation.

By extrapolating the periodic behavior back to $L=0.0$ $0.5 \mu \mathrm{m}$ in Fig. 5, the lower switching field is expected where DWs take the path along the upper branch. This is in agreement with the injection of an initial up chirality DW from the down hook structure with a clockwise rotating field. Over these short distances the DW-vertex interaction takes place before the DW can undergo any Walker breakdown transitions and hence the DW retains its upward chirality and is expected to take the upper branch at the vertex.

In Fig. 5 it is interesting to note that the periodic behavior differs between the two sample thicknesses. While the 20-nmthick structures show reversal at either $H_{C 1}$ or $H_{C 2}$, the majority of hysteresis loops for the 15-nm-thick structures show an average of magnetization reversal events at both $H_{C 1}$ and $H_{C 2}$ and the periodicity is found from the few points showing reversal at either $H_{C 1}$ or $H_{C 2}$. The greater variation in the DW propagation direction for the 15-nm-thick structure indicates either a reduction in the chirality selectivity at the vertex or an increased variation in the Walker breakdown processes during DW propagation along the horizontal bar.

For the extremes of the length scales there is some deviation from this periodic behavior. In the short length regime, this may be associated with an initial DW-vertex interaction before the DW has completely depinned from the hook structure. It may also represent complex dynamical behavior associated with the acceleration of the DW before the regular Walker breakdown cycle is fully established. For larger lengths, slight variations in the injection field and Walker breakdown process may lead to a significant difference in the position through the Walker breakdown cycle after the DW has traveled a significant distance. Therefore it is expected that these changes are averaged out removing the periodic behavior from these measurements for longer length wires.

In Fig. 5(b) $H_{C 1}$ and $H_{C 2}$ are significantly larger than $H_{\mathrm{INJ}}$. This implies that the DW propagates along the horizontal bar and becomes pinned at the vertex before depinning at the greater field, $H_{C 1}$. However, in Fig. 5(a), $H_{C 1}$ and $H_{\mathrm{INJ}}$ are comparable, suggesting that the incident DW from the horizontal bar can propagate through the vertex as it is already propagating above $H_{C 1}$. In this case the small variation in the geometry of these structures has led to a significant difference in the DW-vertex interaction behavior.

These fields found in the experimental measurements are still lower than those predicted by the micromagnetic simulations in Fig. 4. Some of this discrepancy may be accounted for as simulations performed at zero temperature are known to overestimate reversal fields and other differences could arise due to differences in sample geometry and material parameters. Additionally the simulations performed with a two-dimensional mesh represent only a 10-nm-thick structure which could result in systematic differences from the experimental results. However, the difference in interaction strength between a propagating DW at a vertex and the depinning of a DW from a vertex is more likely to be significant.

Further simulations, taking into account the dynamic effects of the incident DW have also been performed. Here, DWs travel towards the vertex where the DW-vertex interaction is investigated as a function of the applied field and the initial DW-vertex separation. During this propagation the micromagnetic structure of the wall transforms between up and down chiralities through a continuous process involving the traverse of an antivortex core through the wall structure. The interaction of these intermediate structures with the 


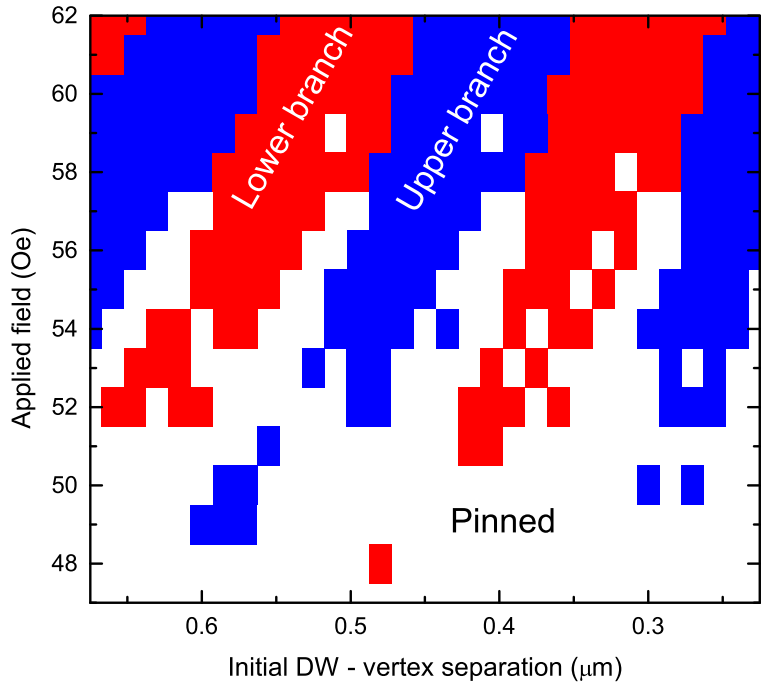

FIG. 6. (Color online) Micromagnetic simulations showing the final state of a dynamic DW-vertex interaction as a function of applied field and initial DW-vertex separation. Regions in white show where the DW becomes pinned at the vertex while red and blue show where the DW propagates along the lower and upper branch, respectively.

vertex leads to a complex interaction where the outcome is summarized in Fig. 6. The final state of the system shows that the DW either becomes pinned at the vertex at low fields (white) or propagates along either the upper (blue) or lower (red) branches at higher fields. At these higher fields the DW alternates between taking the upper and lower path due to Walker breakdown modifications to the DW chirality. The figure also shows that regions of similar behavior occur at shorter DW-vertex separations as the applied field is increased. This represents changes in the average DW velocity and the Walker breakdown periodicity associated with the larger fields.

At low fields the Zeeman energy supplied by the field is insufficient to overcome the repulsion between the magnetically charged DW and vertex. In this case the DW remains pinned in the horizontal nanowire and the magnetization in neither of the two branches is reversed. With larger fields the greater Zeeman energy allows the DW to propagate through the vertex overcoming this pinning effect. In this regime, Fig. 6 shows that the path taken by the DW alternates between the upper and the lower branch, supporting our understanding of this system from the experimental results.

The boundary separating the pinned and propagating regimes also shows a periodic dependence where an increased pinning effect is found, associated with the transition between where the DW takes the upper or lower branch in Fig. 6. This suggests variations in the DW-vertex interaction strength that depend on the different DW micromagnetic structures that emerge throughout the Walker breakdown cycle. This variety of micromagnetic structures has been probed by varying the propagation distance between the initial DW and the interacting vertex. The boundary between the pinned and propagating regimes also occurs at a field significantly lower than the field required to depin a DW from an equivalent vertex shown in Fig. 4. This suggests that the DW-vertex interaction is more complex than our simplistic understanding using the Zeeman field energy to overcome an intrinsic energy barrier. Additional effects relating to the micromagnetic structure of the DW during the interaction become increasingly important in understanding these processes in the dynamic regime.

\section{CONCLUSIONS}

The magnetization behavior of DWs at nanowire vertices has been investigated through both experimental and micromagnetic approaches. The behavior of these DWs is governed by the interactions between the DW micromagnetic spin structure and the spin structure that exists due to the magnetic frustration at the vertex.

The micromagnetic structure of a domain wall contains spins perpendicular to the wire axis which gives it intrinsic asymmetry known as the chirality of the DW. This asymmetry leads to a bias in the DW propagation path that a DW takes as it depins from the vertex. However, as dynamic effects associated with DW motion are considered, modifications to the DW micromagnetic structure, including time-dependent changes, take place. A periodic dependence to the DW path at the vertex arises due to the alternating chirality of the DW from the Walker breakdown process.

The analysis here also suggests further complexity to the DW-vertex interaction beyond a simple depinning model. Although the depinning field from a DW pinned at the vertex is large, DWs propagating towards a vertex can propagate through the vertex at a field below the depinning field. This is associated with differences in the micromagnetic structure of the propagating DWs.

The results demonstrated here build on our understanding of DWs and how their modes of propagation may lead to complex interactions with vertices. However, a full understanding of the behavior of an artificial spin ice system must also consider the way in which multiple vertices are combined to form the structure. Here the initial DW structure was controlled but in these structures the DW in any nanowire will have originated from a previous interaction in a neighboring vertex. Variations in the initial micromagnetic structure of the DW will combine with the interesting time-dependent changes in DW structure and chirality investigated here which will add further complexity to the system.
[1] D. A. Allwood, G. Xiong, C. C. Faulkner, D. Atkinson, D. Petit, and R. P. Cowburn, Science 309, 1688 (2005).

[2] S. S. P. Parkin, M. Hayashi, and Luc Thomas, Science 320, 190 (2008).

[3] P. Vavassori, V. Metlushko, B. Ilic, M. Gobbi, M. Donolato, M. Cantoni, and R. Bertacco, Appl. Phys. Lett. 93, 203502 (2008).
[4] M. Donolato, P. Vavassori, M. Gobbi, M. Deryabina, M. F. Hansen, V. Metlushko, B. Ilic, M. Cantoni, D. Petti, S. Brivio, and R. Bertacco, Adv. Mater. 22, 2706 (2010).

[5] E. Rapoport and G. S. D. Beach, Appl. Phys. Lett. 100, 082401 (2012). 
[6] G. Ruan, G. Vieira, T. Henighan, A. Chen, D. Thakur, R. Sooryakumar, and J. O. Winter, Nano Lett. 10, 2220 (2010).

[7] A. Torti, V. Mondiali, A. Cattoni, M. Donolato, E. Albisetti, A. M. Haghiri-Gosnet, P. Vavassori, and R. Bertacco, Appl. Phys. Lett., 101, 142405 (2012).

[8] E. Rapoport and G. S. D. Beach, Phys. Rev. B 87, 174426 (2013).

[9] Y. Nakatani, A. Thiaville, and J. Miltat, J. Magn. Magn. Mater. 290-291, 750 (2005).

[10] M. Klaui, H. Ehrke, U. Rudiger, T. Kasama, R. E. DuninBorkowski, D. Backes, L. J. Heyderman, C. A. F. Vaz, J. A. C. Bland, G. Faini, E. Cambril, and W. Wernsdorfer, Appl. Phys. Lett. 87, 102509 (2005).

[11] D. M. Burn, E. Arac, and D. Atkinson, Phys. Rev. B 88, 104422 (2013).

[12] E. R. Lewis, D. Petit, A-V. Jausovec, L. O'Brien, D. E. Read, H. T. Zeng, and R. P. Cowburn, Phys. Rev. Lett. 102, 057209 (2009).

[13] L. O’Brien, A. Beguivin, D. Petit, A. Fernandez-Pacheco, D. E. Read, and R. P. Cowburn, Philos. Trans. R. Soc., A 370, 5794 (2012).

[14] P. Mellado, O. Petrova, Y. Shen, and O. Tchernyshyov, Phys. Rev. Lett. 105, 187206 (2010).

[15] A. Pushp, T. Phung, C. Rettner, B. P. Hughes, S-H. Yang, L. Thomas, and S. S. P. Parkin, Nat. Phys. 9, 505 (2013).

[16] S. Ladak, D. E. Read, T. Tyliszczak, W. R. Branford, and L. F. Cohen, New J. Phys. 13, 023023 (2011).

[17] S. Ladak, D. E. Read, G. K. Perkins, L. F. Cohen, and W. R. Branford, Nat. Phys. 6, 359 (2010).
[18] K. Zeissler, S. K. Walton, S. Ladak, D. E. Read, T. Tyliszczak, L. F. Cohen, and W. R. Branford, Sci. Rep. 3, 1252 (2013).

[19] M. Hayashi, L. Thomas, Ya. B. Bazaliy, C. Rettner, R. Moriya, X. Jiang, and S. S. P. Parkin, Phys. Rev. Lett. 96, 197207 (2006).

[20] L. Thomas, R. Moriya, C. Rettner, and S. S. P. Parkin, Science 330, 1810 (2010).

[21] M. Hayashi, L. Thomas, C. Rettner, R. Moriya, Ya. B. Bazaliy, and S. S. P. Parkin, Phys. Rev. Lett. 98, 037204 (2007).

[22] N. L. Schryer and L. R. Walker, J. Appl. Phys. 45, 5406 (1974).

[23] G. S. D. Beach, C. Nistor, C. Knutson, M. Tsoi, and J. L. Erskine, Nat. Mater. 4, 741 (2005).

[24] D. M. Burn and D. Atkinson, Appl. Phys. Lett. 102, 242414 (2013).

[25] E. R. Lewis, D. Petit, L. O’Brien, A-V. Jausovec, H. T. Zeng, D. E. Read, and R. P. Cowburn, Appl. Phys. Lett. 98, 042502 (2011).

[26] E. R. Lewis, D. Petit, L. Thevenard, A-V. Jausovec, L. O’Brien, D. E. Reard, and R. P. Cowburn, Appl. Phys. Lett. 95, 152505 (2009).

[27] M. Donahue and D. Porter, The Object Oriented MicroMagnetic Framework (OOMMF) Project at ITL/NIST. The OOMMF code is available at http://math.nist.gov/oommf.

[28] J. Fidler, J. Phys. D: Appl. Phys. 33, R135 (2000).

[29] R. D. McMichael and M. J. Donahue, IEEE Trans. Magn. 33, 4167 (1997).

[30] O. A. Tretiakov, D. Clarke, G.-W. Chern, Ya. B. Bazaliy, and O. Tchernyshyov, Phys. Rev. Lett. 100, 127204 (2008). 